\title{
A BOOK RETRIEVAL AND LOCATION SYSTEM BASED ON REAL-SCENE 3D
}

\author{
Wei Shuangfeng ${ }^{1,2,3,4},{ }^{1}$ Li Boyi $^{* 1}$, Guo Zhihao ${ }^{1}$, Guo Shuai ${ }^{1}$,Cheng Liurun ${ }^{1}$ \\ ${ }^{1}$ School of Geomatics and Urban Spatial Informatics, Beijing University of Civil Engineering and Architecture, Beijing, 102616 \\ ${ }^{2}$ Engineering Research Center of Representative Building and Architectural Heritage database, Ministry of Education, Beijing, \\ 102616 \\ ${ }^{3}$ Key Laboratory for Urban Geomatics of Ministry of Natural Resources, Beijing, 102616 \\ ${ }^{4}$ Beijing Key Laboratory for Architectural Heritage Fine Reconstruction \& Health Monitoring, Beijing, 102616
}

KEY WORDS: Trolley SLAM, IndoorViewer, POI, API, Book Retrieval

\begin{abstract}
:
With the development of urbanization, the building structures are more and more complex, and various moving objects, such as human beings, robots and unmanned aerial vehicles, often travel through indoor and outdoor 3D space, which puts forward higher requirements for the accurate search and location in indoor and outdoor space. At present, most of the spatial location methods for indoor entities are carried out through 2D maps. However, the indoor environment is a complex 3D space, which increases the difficulty of the search process. In addition, 2D map cannot accurately display the 3D spatial position of the entity. Therefore, it is difficult for 2D maps to search and locate in complex environment. Therefore, how to quickly and effectively carry out spatial location query in complex indoor environment has become an urgent problem to be solved. Taking the library of Beijing University of Civil Engineering and Architecture as an example, this paper obtains the indoor 3D information of the library based on SLAM, processes and publishes the acquired 3D information on IndoorViewer, and uses its API in the book retrieval system. Finally, a book retrieval and location system based on real-scene 3D is finished.
\end{abstract}

\section{INTRODUCTION}

With the development of mobile internet technology and the maturation of indoor positioning technology, people's demand for navigation applications has expanded from the original outdoor navigation to the integrated navigation inside and outside the room. The way to locate and navigate to the target accurately in the complex interior $3 \mathrm{D}$ environment has become an urgent problem to be solved in today's society.Though 2D map is still the main way of indoor and outdoor navigation and location, its drawbacks such as poor map representation and augmented reality, simple model, lack of intuition, and inaccurate positioning for 3D target are particularly prominent in complex indoor space.There is no doubt that 2D navigation cannot meet the actual positioning needs of various moving objects, such as human, unmanned aerial vehicles and robots, and cannot apply to the increasingly complex indoor space (WEI Shuangfeng etc al. 2018) . 2D maps cannot accurately represent the location of the target in the complex 3D space. It is difficult to satisfy the user's spatial search and location requirements in complex environments.

Considering the contradiction between the rapid development of the application of GIS from outdoor to indoor and the lag of theory and technology methods (WU Wei, 2012), this paper takes the library of Beijing University of Civil Engineering and Architecture as an example, aiming at the difficulty for teachers and students to find books in the library, used SLAM technology to acquire the indoor spatial data of the library, including point cloud data and $3 \mathrm{D}$ real scene images. The acquired data are processed in IndoorViewer, POI are established for the bookshelves of the library, and the spatial location of books is determined according to the corresponding relationship between books and bookshelves, so as to realize the function of path planning. In the book retrieval system, API interface of IndoorViewer is used to realize a book retrieval and positioning system based on 3D real scene.

\section{PRINCIPLES AND METHODS}

\subsection{Data acquisition method based on trolley SLAM}

SLAM (Simultaneous Localization and Mapping), also known as CML (Concurrent Mapping and Localization), is a kind of synchronous positioning and mapping technology commonly used in robots. On the one hand, it relies on the created map information for self-positioning. On the other hand, it updated the map according to the positioning results ( LIANG Mingjie et al. 2013 ). When the robot is in an unknown environment, it can use SLAM to move while drawing a complete map of the environment, so that it can travel through the indoor environment effectively. In terms of indoor 3D, SLAM can be applied to acquire indoor 3D information (YU Jianwei et al. 2016 ) : Scanning indoor space information by trolley SLAM (HUITL R et al. 2013) can obtain high-precision 3D real scene images and point cloud data. The SLAM system of cart uses Gmapping algorithm (GRISETTI G et al. 2007), which combines vertical $2 \mathrm{D}$ laser scanner to obtain indoor $3 \mathrm{D}$ information. The Gmapping algorithm combines high-precision wheel ranging information. The experimental results show that the pose error is less than $2 \mathrm{~cm}$ (Wei Shuangfeng et al. 2018 ). This paper used the NavVhis M3 scanning vehicle (Figure 1) as the scanning tool to collect indoor 3D information in the library of Beijing University of Civil Engineering and Architectural.

2.1.1 Scanning principle : In this experiment, the NavVis M3 Indoor scanning vehicle is used as the scanning tool. It is equipped with six cameras with 16 million pixels, a horizontal laser scanner and two vertical laser scanners ( HU Bo et al. 2019). During the scanning process, the scanner takes six

\footnotetext{
* Corresponding author
} 
images after moving on for a while, and the images are stitched together to achieve the synthesis of $3 \mathrm{D}$ real scene images. In the course of the journey, the laser scanner can capture within 30 meters and $270^{\circ}$ range of the scene by continuous scanning, in order to achieve full access to the indoor $3 \mathrm{D}$ information, and use the location algorithm for the obtained data to locate themselves and provide the required data of map construction and then generate point cloud data.

The obtained data can be processed to produce 3D real scene map and indoor 2D floor plan. The obtained point cloud data is available for spatial analysis and modeling (Hua Tai Tian $\mathrm{Yu}$ ).

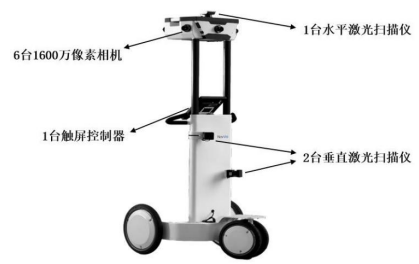

Figure 1. NavVis M3 Indoor scanning vehicle

2.1.2 Scanning method: The scanning scope of the experiment is the library in Beijing University of Civil Engineering and Architecture, which has a total of 8 floors (including the underground floor). The experiment mainly scanned the first floor, the book storage floor (the second to fifth floor) and the university's history museum (the sixth floor).

Before starting the scanning, it is necessary to plan the scanning path, and make different scanning schemes based on the internal structure characteristics of each floor of the library. The planning of the scanning path requires consideration of the following factors:

1. In order to improve the accuracy of the data, it is necessary to establish a closed loop of the dataset. Part of the path needs to overlap in order to achieve registration by using the route intersections.

2. Because of the variable character of the indoor environment, for example, the adjustment of the library tables distribution and chairs will change the spatial layout, in order to reduce the workload of future re-scanning, scanning should be subdivided.

3. Before scanning, it is necessary to measure the width of the indoor channel to confirm whether the scanner can pass, which provides a reference for scanning path planning.

4. Scanning needs to be done floor by floor, when a floor scan is completed,scanning should be over. The scanning vehicle should be moved to the next target floor.

5. The maximum time for a single scan is 45 minutes, otherwise the best effect cannot be achieved.

After starting the scanner and setting the relevant parameters, you need to position it : rotate the cart slowly for two laps to get its own position. During the scanning process, the cart needs to be moved at a slow speed (no more than $6 \mathrm{~m} / \mathrm{s}$ ), and the speed is maintained evenly. Every 1 meter of travel, the cart beeps, then the cart should be stopped and take images of the surroundings. During the scanning process, you need to pay attention to the following issues:

1. The trolley can not travel too fast, so as not to affect the quality of the images.
2. The cart needs to be kept at a distance of about 1 meter from the surrounding wall, otherwise the image will not work effectively. Too far distance from the wall will reduce the fineness of the image (HU Bo, 2018).

3. While scanning in the broad space, the route should travel according to the planning ranks. In addition, while walking along the closed-loop route, laser scanner should avoid losing reference points.

4. Get high-quality three-dimensional images at the corner of the route by manually shooting for multiple times. 5. Pay attention to scan the elevator and connect the upper and lower floors near the ladder, to easily build a navigation network.

6. When encounter obstacles that lead to not pass properly, you should end this scan and start again after passing.

7. During the scanning process, if there are pedestrians moving nearby, pause the scan, wait for pedestrians to pass before continuing.

8. If the scanning person is high, be careful not to take yourself into its sensors.

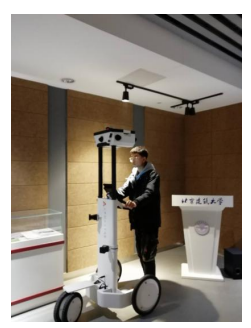

Figure 2. Scanning process

\subsection{IndoorViewer based on the data processing}

Based on the SLAM Trolley technology, this article obtains the spatial data of the library, including laser scanner data and realscene images. Next, you need to use NavVis software to process data: First, the original data, published data and network data need to be preprocessed, and then in the IndoorViewer ,carrying out the registration of Raw point cloud data (relative registration of data units and global registration), followed by we create navigation diagrams and cloud maps of IndoorViewer in order to facilitate subsequent indoor path navigation, and finally upload the above processed data to the network side and publish.

2.2.1 About IndoorViewer: IndoorViewer is NavVis ' browser-based application for visualization, digital enrichment and navigation of indoor spaces. Core features of the web-based IndoorViewer client are:

1. Browsing $360^{\circ}$ panorama views of an indoor space mapped with NavVis technology.

2. Browsing, creating, and editing points of interest (POI): right-click anywhere to create a POI in 3D space.

3. Route planning in $3 \mathrm{D}$ between any locations in a NavVis-mapped indoor space.

4. Point cloud display and point cloud alignment tools for dataset organization.

IndoorViewer helps users get information about public places and business spaces and get a first impression, and users can search for indoor destinations in advance and get a detailed walking direction in the interior space. 
InDoorViewer Based on the most advanced HTML5 and WebGL technology, which is available at all Web Browsers and could run in a variety of desktops and mobile systems, with good compatibility.

2.2.2 Data preprocessing: Data preprocessing is mainly divided into raw data preprocessing, network data preprocessing and publishing data preprocessing. Between them, raw data preprocessing requires working with all raw data in the target directory and to preserve the results; and start post-processing of dynamic object deletion on all recorded datasets, which called Navvisstart-postprocessing.sh, and use--Filter-dynamicobjects to delete objects moved during a scan (Pedestrians walking around). Publishing data preprocessing and network data preprocessing is mainly to create an instance of the current project and load the scanned data unit into the instance.

After the instance is registered and logged in, the interface of IndoorViewer displays a number of administrative icons, clicking on the management settings and it will display the actual area properties of the current instance. At this point, the data preprocessing work is complete.

2.2.3 Point cloud registration: The aim of Point Cloud Registration is to make the point Cloud dataSet to match and associate with the formation of Indoor space between the floors, between indoor and outdoor, and between its own geographical location and the global map's correct location association including relative registration of data units (Datasets Alignment) and global Registration (Geo-registration) .

Relative registration: In the IndoorViewer, opening the registration interface. It will be divided into amplitude , displaying out of four pieces of pictures (building top view, building point cloud data, a side view from north to south and a side view from east to west). First, a data unit is specified as a reference data unit, so that all data units rotate at the starting point of the selected unit as the origin, using theTransform Angle rotation and axial panning options in menus, combined with four view images. Precise point cloud registration. In this article, the elevator wells and stairs of each floor are used as reference for registration and splicing, and the projection overlap degree of each layer in the top view is checked, and the relative registration of each data unit in the library is completed by careful verification and comparison.
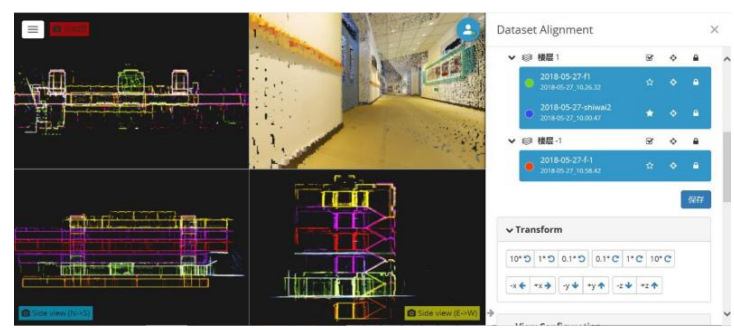

Figure 3. Relative registration of data unit

Global registration: It is based on OSM (Open Street Map), the main purpose of the location registration method is to set the located position under the global coordinate system to pave the groundwork for subsequent generation of navigation diagrams and cloud maps. Because of the defects in the degree of refinement of OSM, with the help of Google Maps and other third-party platforms for auxiliary positioning[11]: According to Google MapsFor a comparison reference, we drag and drop the previously completed relative registration of the data unit set
toOSMIn the corresponding geographic location, the matching point cloud is shown in the figure:

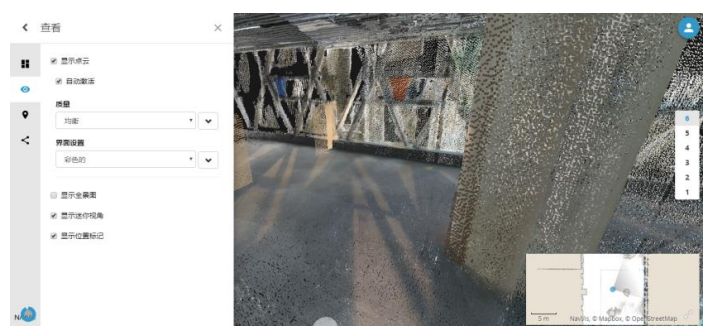

Figure 4.Point cloud after registration

2.2.4 Create navigation diagrams and cloud maps:There are two types of maps are displayed in the interface of IndoorViewer: navigation diagrams and cloud maps. Between them, the navigation diagram shows the possible path network to a position in the way of road network and three-dimensional reality.Cloud Map is a two-dimensional floor plan of the scanning area to understand the plane range of the building.

This article uses the registration parameter file obtained by the previous point cloud registration to create a navigation chart and a cloud map.

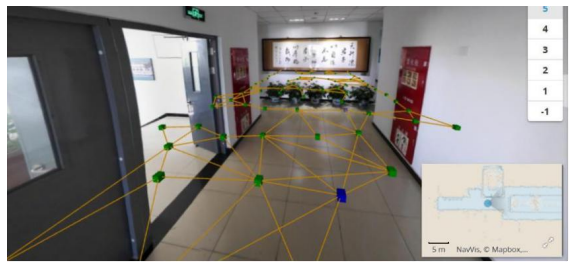

Figure 5. Navigation map after registration

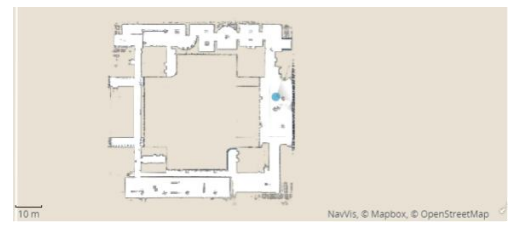

Figure 6.Cloud map after registration

2.2.5 Data publishing: After completing the navigation diagram and cloud map creation, you need to upload data to the network server and publish it. The steps are as follows:

1. Log on to the server and create a project directory on the network server.

2. Return to under native command, or reopen a command window.

3. Use rsync command to transmit all data files and Xml files.

4. Create IndoorViewer instances on a network server and publish the data.

The results of the network release of the final indoor threedimensional reality map are shown in the figure: 


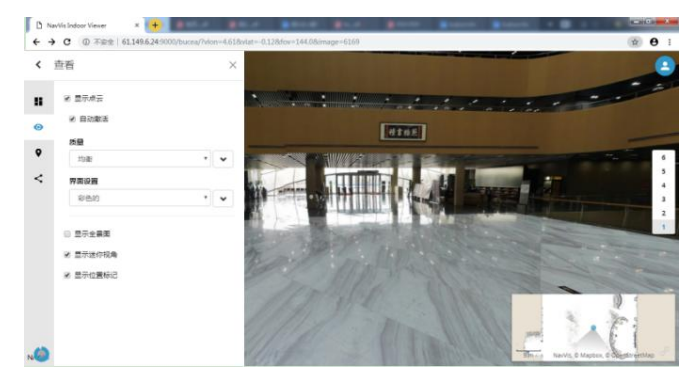

Figure 7. Results of live map release

\subsection{The Indoor path planning based on IndoorViewer}

2.3.1 Add points of interest: Points of Interest (POI) is a term in a geographic information system that generally refers to all geographical objects that can be abstracted as points, especially which are closely related to people's lives, such as stepping ladders, bookshelves in libraries, toilets and so on. Each points of interest contains four parts :name, category, coordinates, category. The main purpose of the point of interest is to describe the address of a thing or an event, which can greatly enhance the ability to describe the location of a thing or an event and the ability to query, and improve the accuracy and speed of geographical positioning.

This paper adds points of interest to the main feature points in the library (elevators, ladders, bookshelves, toilets, books and periodicals self-service lending machines). Among them, the bookshelf point of interest to add work is very important: the database of the book retrieval system provides books, categories, corresponding bookshelves and other information, in order to find out the space location of the book in the IndoorViewer, you need to add points of interest to each layer of the bookshelf and add relevant information to achieve the spatial positioning of the book.

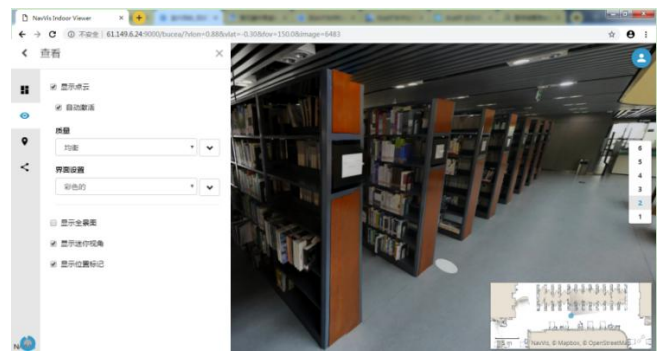

Figure 8. Adds POI to the bookshelf

In the IndoorViewer, we can right-click the target position in the $3 \mathrm{~d}$ real scene map and select "create interest point". The interface for editing the interest point is shown in the figure below:

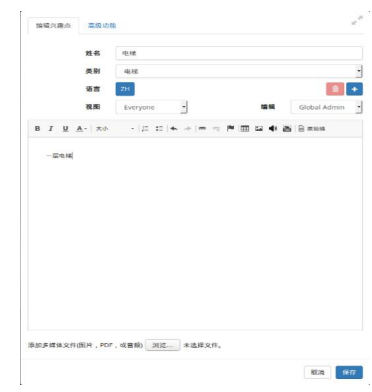

Figure 9. Interest Point Editing Interface

The steps for creating POI are as follows:

1. Input the English name of the POI;

2. Select categories for newly created POI;

3. Add a description to the text box;

4. Select permission group;

5. Click "save".

Through the function of adding POI of Indoor Viewer, this paper adds POI to the bookshelves, elevators, stairs, toilets and other features of the library of Beijing University Of Civil Engineering And Architecture, which establishes the spatial correspondence relationship and lays the foundation for the follow-up path planning work.

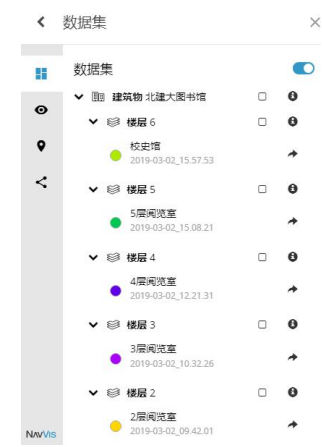

Figure 10. Created data sets and POI

2.3.2 Path planning method: After creating POI, this paper uses the path planning function of Indoor Viewer to navigate the path between two interest points in the library.

After activating the path planning function in the IndoorViewer add-on module, each interest point panel has a "path planning" option.Selecting the corresponding starting point (such as the library gate) and the end point (such as the bookshelf), the system automatically calculates and generates the planning path and related navigation information according to the navigation node network, and uses the direction arrows associated in turn to represent the navigation path in the three-dimensional real map; and the two-dimensional plane map in the lower right corner also shows the navigation path, giving users a more intuitive two-dimensional and three-dimensional linkage.In addition, the path planning panel in the upper left corner clearly shows the distance between the two POI and the time required to walk.

Through the function of path planning, users can plan and navigate the path in the three-dimensional scene and cloud map from their own location to the bookshelf corresponding to the book as the end point, and obtain information such as the distance between the starting and ending points and the time required to reach the end point, so as to obtain more effective indoor space navigation assistance. 


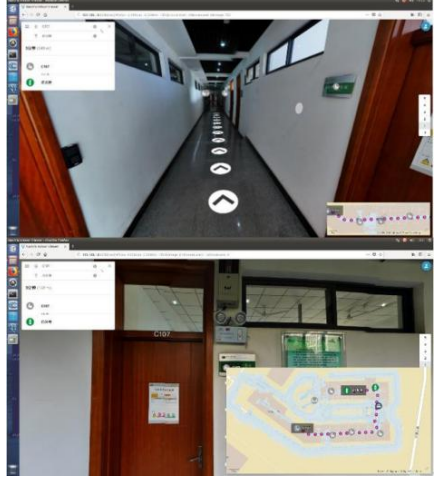

Figure 11. Path planning in Indoor Viewer

\subsection{Calling IndoorViewer API interface in the book retrieval system}

API (Application Programming Interface) is a set of predefined functions designed to provide applications and developers with the ability to access a set of routines based on certain software or hardware without access to source code or understanding the details of internal working mechanisms.

In this paper, two methods of inserting the Indoor Viewer API into the book retrieval system are designed with IFrame and Javascript respectively, and a comparative study is made on them.

1. Embedding with IFrame: Embedding IndoorViewer with IFrame HTML elements is the easiest way.IFrame can be freely located on embedded pages, just like a separate website. When embedded in IFrame, the Indoor Viewer can be displayed as a window in the view. The effect is shown as follows:

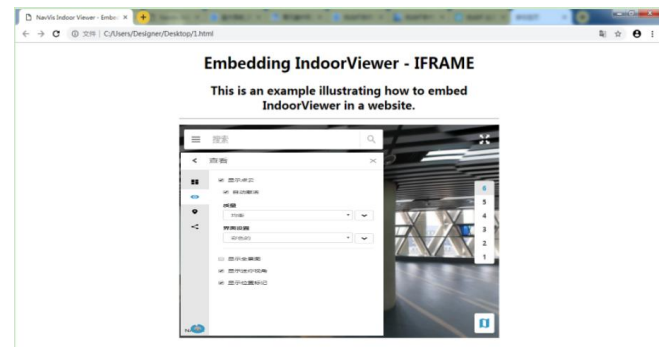

Figure 12. Implementing the effect by invoking API with IFrame

The limitations of the IFrame method lie in:

(1) Embedded Web pages cannot receive feedback from IndoorViewer (for example, when changing location or Open a POI.

(2) The user interface of IndoorViewer is limited by the size of IFrame.UI elements should not exceed the boundaries of IFrame.

(3) Only through client configuration parameters can the appearance of user interface elements of IndoorViewer be changed. Custom CSS and HTML can not be used for advanced customization.

(4) Cannot use JavaScript for behavior customization, such as custom search providers;

(5) The URL of the embedded page will not change as the user moves to IndoorViewer.
2. Embedding with JavaScript API: Embedding IndoorViewer with JavaScript API provides great flexibility for embedding it into the target website.In this way, the library retrieval system does not need to be hosted in the same domain as the IndoorViewer, only need to include and create an IndoorViewer object.

Finally, this paper realizes a book retrieval and positioning system based on three-dimensional real scene. The system can achieve the following functions:

1. After users enter the system, they can retrieve books through the input box provided by the system. The retrieval system judges the books they need by identical or similar labels, and calculates the similarity of users by identical labels, so as to realize the book recommendation function: if the books that users need are not found or the books have been borrowed, the relevant recommended books will be displayed (Xu Yaru, 2018).

2. After finding the books that users need, the system obtains the corresponding bookshelves through the database, and invokes the Indoor Viewer interesting point query function to obtain the spatial location of the bookshelves, and then combines the path planning function with indoor positioning technology to implement navigation for users.

3. The user can switch the real image by clicking on the direction arrow of the three-dimensional real map. In addition, the user can click on the position of the twodimensional plane map to complete the switching of the three-dimensional real image. The user can combine the three-dimensional real image with the two-dimensional plane map to get the position of the bookshelf where the book is located, thus realizing the integration of the threedimensional real scene and the two-dimensional map.

4. Combining the three-dimensional image and twodimensional plan, it provides the roaming function of the library. Users can know the distribution of Library books, the functional zoning of each floor, the location of toilets and stairwells through the mobile terminal.

5. Provide an administrator account to manage users, books and navigation paths. If the elevator fails to work, the administrator can set the elevator as an obstacle in the navigation path. The system will bypass the obstacles and find alternative routes in the path analysis.

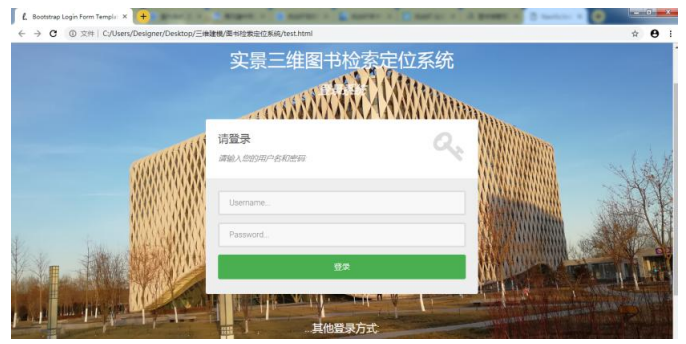

Figure 13. Real-scene three-dimensional book retrieval system

\section{CONCLUSION}

Aiming at the problem of "difficult to find books" in libraries, combined with the hotspot of indoor navigation and positioning technology, this paper obtains the three-dimensional spatial data of the library of Beijing University Of Civil Engineering And Architecture by using SLAM technology of carts, preprocesses 
the data, registers point cloud and global registration, generates navigation map and cloud map, and publishes them on the network; it is important for libraries including bookshelves in Indoor Viewer.The feature adds interest points and realizes the function of path planning by expanding module. By calling the API interface of IndoorViewer, the function of IndoorViewer is embedded in the book retrieval system, and the book retrieval and positioning system based on three-dimensional scene is realized.

Compared with other book retrieval systems, this paper uses three-dimensional scene to locate books for the first time, expanding the location of books and periodicals from twodimensional plane map to three-dimensional space, enabling users to search books quickly and effectively in the complex indoor environment of the library; the linkage and switching of three-dimensional and two-dimensional plane map, the combination of three-dimensional scene and navigation semantic network also make queries. The accuracy and intuition of the results have been greatly improved, and a more friendly user experience has been created.

This paper has broad application prospects for the design method of real-scene three-dimensional retrieval and positioning system: it combines with indoor navigation technology, achieves real-time navigation function by acquiring the location of users; applies to large public places such as shopping malls, airports, stations, etc., to provide services for target location query in complex space; realizes the integrated indoor and outdoor navigation of intelligent robots and UAVs, and promotes it.The development of logistics, transportation and emergency rescue.

\section{REFERENCES}

WU Wei. Modeling method of indoor three-dimensional navigation in shopping malls [D]. Nanjing Normal University, 2012.

WEI Shuangfeng, HU Bo, ZHANG Xiaoyu, etc al. Integrated three-dimensional navigation path planning for indoor and outdoor digital campus [J], Surveying and Mapping Bulletin, $2018(10)$

LIANG Mingjie, MIN Huaqing, LUO Ronghua. Summary of simultaneous localization and map creation based on graph optimization [J], Robot ,2013, 35 (4).

YU Jianwei, WEI Chi. Indoor mobile measurement system based on SLAM and its application [J]. Surveying and mapping bulletin, 2016 (06): 146-14

HUITL R, SCHROTH G, HILSENBECK S, et al. TUMindoor: an extensive image and point cloud dataset for visual indoor localization and mappng [C]//IEEE International Conference on Image Processing. Orlando: IEEE, 2013.

GRISETTI G, STACHNISS C, BURGARD W. Improved techniques for grid mapping with rao-blackwelled particle filters [J]. IEEE Transactions on Robotics, 2007, 23 (1): 34-46.

WEI Shuangfeng, LIU Zhenbin, ZHAO Jianghong, etc. Summary of SLMA Indoor 3D Reconstruction Technology [J], Surveying and Mapping Science, 2018 (07).
HU Bo, WEI Shuangfeng, YAN Qiang. SIAM indoor real scene three-dimensional mapping and its application [J]. Surveying and mapping bulletin, 2019 (01).

Hua Tai Tian Yu .http://www.wtechgnss.com/

HU Bo. Indoor scene three-dimensional mapping based on SLAM trolley [D], Beijing University of Architecture, 2018.

XU Yaru. Design and Research of Label-based Personalized Book Recommendation System [D], Shandong Normal University, 2018. 